\title{
ULTRAFAST CARRIER DYNAMICS IN GaAs NANOWIRES
}

\author{
V.N. Trukhin ${ }^{\text {a,c }}$, A.D. Buravlev ${ }^{\text {a,b }}$, V. Dhaka ${ }^{\text {d }}$, G.E. Cirlin ${ }^{\text {a,b }}$, I.A. Mustafin ${ }^{\text {a,c }}$, \\ M.A. Kaliteevski ${ }^{\text {a,b }}$, H. Lipsanen ${ }^{\text {d }}$, and Yu.B. Samsonenko ${ }^{\text {a,b }}$ \\ ${ }^{a}$ Ioffe Physical Technical Institute, Politekhnicheskaya 2, 194021 St. Petersburg, Russia \\ E-mail: valera.truchin@mail.ioffe.ru \\ ${ }^{\mathrm{b}}$ St. Petersburg Academic University, Khlopina 8/3, 194021 St. Petersburg, Russia \\ 'NRU ITMO, Kronverkskiy 49, 197101 St. Petersburg, Russia \\ ${ }^{\mathrm{d}}$ Aalto University, Lämpömiehenkuja 2, 02150 Espoo, Finland
}

Received 18 November 2013; accepted 4 December 2013

\begin{abstract}
The results of investigation of electronic transport in GaAs nanowires (NWs), which were grown by MBE and MOCVD on n-type GaAs(111) substrates are presented. The time-resolved dynamics of photocarriers were studied by opticalpump terahertz generation-probe time-domain spectroscopy. The dynamics of THz generation for different levels of excitation for the GaAs NWs grown by MBE can be explained by separation of photoinduced carriers in the contact field with subsequent screening of the contact field and with non-saturable carrier recombination. The dynamics of $\mathrm{THz}$ generation for the GaAs NWs grown by MOCVD is due to the growth of the mobility of carriers as they are being grabbed to the non-radiative centres and as a consequence of carrier retrapping. The shift of $\mathrm{THz}$ pulse is connected with the changes of a phase in transmission and reflection $\mathrm{THz}$ pulse through the border between the absorbing and non-absorbing medium.
\end{abstract}

Keywords: THz, nanowires, electronic transport, non-radiative centres

PACS: 78.67.Uh, 71.55.Eq, 72.20.-I, 72.20.Jv

\section{Introduction}

Free-standing semiconductor nanowires (NWs) are among the most promising one-dimensional nanoobjects for applications in nanoelectronics and nanophotonics. Accordingly, a study of the fundamental electron transport properties of NWs is a priority. Terahertz generation is very sensitive to carrier density and mobility, thus terahertz time-domain spectroscopy is a promising tool for obtaining information concerning the ultrafast carrier dynamics in NWs. Therefore, a study of the generation of terahertz radiation in semiconductor NWs by creating a non-equilibrium electron-hole plasma will be quite a successful method for studying transport, relaxation, and recombination properties of electrons in NWs. This paper is devoted to the investigation of electronic transport in GaAs NWs, which were grown using molecular beam epitaxy (MBE) and metal-organic vapour phase epitaxy (MOVPE) on n-type GaAs(111) substrates.

\section{Results}

The NW sample was fabricated using an EP1203 MBE reactor equipped with $\mathrm{Ga}$, As, and Au effusion cells.
The sample was grown by the so-called vapour-liquidsolid (VLS) method, and it has a metal droplet on the top of the NW.

The sample was grown by using $\mathrm{Au}$ as a growth catalyst, a $100 \mathrm{~nm}$ thick GaAs buffer layer was grown on the GaAs(111)B substrate for this sample after the total desorption of an oxide layer at $620^{\circ} \mathrm{C}$. The deposition of a $0.3 \mathrm{~nm}$ thick Au layer was performed to promote the formation of NWs by the growth catalyst (at a temperature of $580^{\circ} \mathrm{C}$ ). Then NWs were grown at the same substrate temperatures. The NWs were undoped, but the NWs grown in such conditions could have had slightly n-type doping with a concentration of the order $10^{16} \mathrm{~cm}^{-3}$. Due to a relatively high substrate temperature, the NW height was $\sim 300 \mathrm{~nm}$ and the diameter $\sim 20 \mathrm{~nm}$. The $\mathrm{Au} / \mathrm{Ga}$ droplet of the sample leads to the formation of the Schottky barriers and therefore to the formation of an associated contact field, as shown in Fig. 1. Note that the direction of the contact field depends on the type of the doping of the NWs.

The growth of the samples was monitored using in situ reflection high-energy electron diffraction.

The used sample was found to have a wurtzite crystallographic phase. 


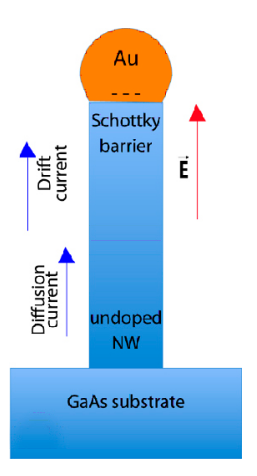

Fig. 1. The scheme of photocurrent in the NW sample.

Photoexcitation of GaAs NWs was performed by Ti:Sapphire lasers operating at a wavelength of $800 \mathrm{~nm}$ with a repetition rate of $78 \mathrm{MHz}$, producing $10 \mathrm{~nJ}$ pulses with the duration of $15 \mathrm{fs}$ and $90 \mathrm{fs}$. In order to detect the $\mathrm{THz}$ emission, electrooptic sampling was used via a $1 \mathrm{~mm}$ thick ZnTe crystal. This provided electric field amplitude and phase information. For the power measurements, a Golay cell detector was used.

To investigate the dynamics of charge carriers we have used two continual optical pulses to excite the photo-induced charge carriers in the NWs and investigate the dependence of $\mathrm{THz}$ power as a function of delay between two pulses. The pulse intensities were chosen to ensure a linear $\mathrm{THz}$ response to the excitation. Both the first and the second excitation pulses have a duration of $90 \mathrm{fs}$. However, the sequence of the "second pulses" was additionally modulated and detection of $\mathrm{THz}$ radiation was synchronized with modulation of the "second pulse". Thus, the influence of the carriers created by the first optical pulse on $\mathrm{THz}$ emission from the carriers created by the second optical pulse moving in the NWs was investigated.

It should be noted that the diameter of GaAs NWs, which were grown by $\mathrm{MBE}$, is less than or equal to $20 \mathrm{~nm}$. The Bohr radius of the exciton $a_{\mathrm{ex}}$ in GaAs is $14 \mathrm{~nm}$ [1]. As long as $d<2 a_{\mathrm{ex}}$, the nature of the electronic states is affected by quantum confinement. Therefore, such nanostructures can exhibit 1D transport properties even at room temperature.

Figure 2 shows the time dynamics of $\mathrm{THz}$ radiation from the moment when electron-hole plasma was excited by the "first optical" pulse. The maximum suppression of $\mathrm{THz}$ power takes place when the delay between optical pulses is 3 ps. This can be explained by the separation of photoinduced carriers in the contact field with subsequent screening of the contact field. As the Schottky barrier depletion region has a length of about 1 micron [2], assuming that the contact field is spreading on the length of the NWs $(300 \mathrm{~nm})$, one can estimate the drift velocity of carriers as $10^{5} \mathrm{~m} / \mathrm{sec}$.

The dynamics of $\mathrm{THz}$ generation for different levels of excitation are presented in Fig. 3. On this figure

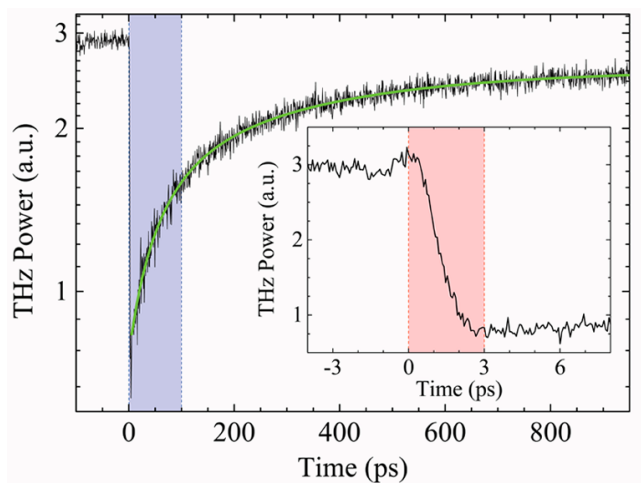

Fig. 2. The measured THz energy as a function of time delay relative to the prepulse for $n$-type GaAs NW. The inset shows this dependence for a small delay value.

one can see the steep drop of $\mathrm{THz}$ field too, the maximal reduction of $\mathrm{THz}$ power occurs when the delay between pulses is about 3 ps. Subsequently, in Fig. 3 one can see the recovery of efficiency of the generation of $\mathrm{THz}$ radiation. At high intensity of the first optical pulse, subsequent restoration of $\mathrm{THz}$ power occurs in two stages. At low intensity of the first optical pulse, the first stage is absent and there is slow recovery of $\mathrm{THz}$ power with a time in the course of $3 \mathrm{~ns}$.

The received results can be explained as follows. The non-equilibrium holes while moving in the contact field are quickly captured by non-radiative centres located on the surface of the NWs (movement of the holes is not one-dimensional). Naturally, the trapped holes will contribute to the reduction in the contact field.

This process certainly prevents holes approach the boundary of the Schottky barrier metal-semiconductor. But at a concentration of non-equilibrium carriers greater than the concentration of non-radiative centres, the holes that are not captured will approach the boundary of the semiconductor-metal and they will be annihilated by electrons from the metal. Thus, the charge of the Schottky barrier will decrease. The sub-

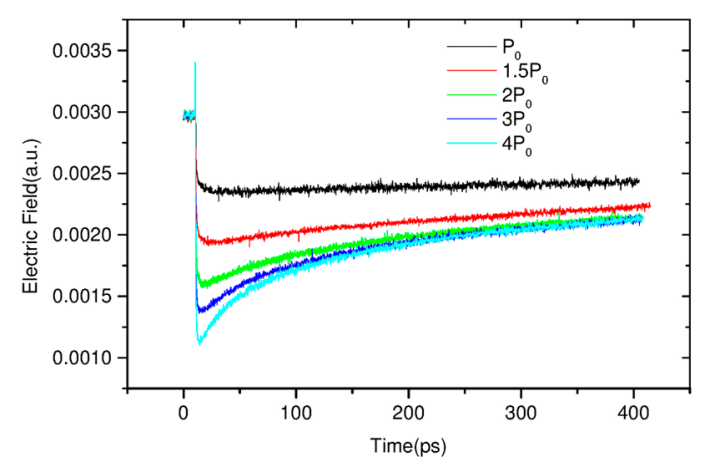

Fig. 3. The measured THz field as a function of time delay relative to a pump pulse $\left(P_{0}=10 \mathrm{~mW}\right)$. 
sequent recovering of the total charge of the Schottky barrier will be due to recharging capacity of the Schottky barrier and it will be determined by the Maxwell relaxation time.

Thus, the fast stage of this restoration is mainly due to the charge trapping, recharging capacity of the Schottky barrier, and appears only at high first-pulse optical intensity and high carrier density. The second stage of this restoration is mainly associated with the carrier recombination. The duration of the first stage is about 72 ps in the case considered in Fig. 2.

It should be mentioned that in the case of charge trapping at the NW's surface and low intensity of the first optical pulse, the initial deep decay of $\mathrm{THz}$ generation would not be observed and the following restoration would be fast. Non-radiative recombination due to charge trapping at the NW's surface reduces the charge carrier lifetime in GaAs NWs to 1 ps [3]. Therefore, charge carrier dynamics observed in our experiments is explained by electron transport in the 1D NW structure.

The dynamics of $\mathrm{THz}$ generating for different levels of excitation for the GaAs NWs with a diameter of about $40 \mathrm{~nm}$ grown by MOVPE are shown in Fig. 4 . Both the first and the second excitation pulses have a duration of $15 \mathrm{fs}$. It has significant differences from the one described earlier. During first several picoseconds there is a small drop, then it grows dramatically for tens of picoseconds and then falls slowly. We assume that this small drop of the energy of $\mathrm{THz}$ radiation is related to the process of screening the contact field by non-equilibrium electrons and holes separating in this electric field. The contact field is formed by forming a Schottky barrier at the contact area between the metal cap of $\mathrm{Au}$ and the semiconductor. Accurately determining the time of the contact field screening is not possible, because at the same time there is a process that leads to an increase in efficiency of the generation of $\mathrm{THz}$ radiation. Increasing efficiency of the generation

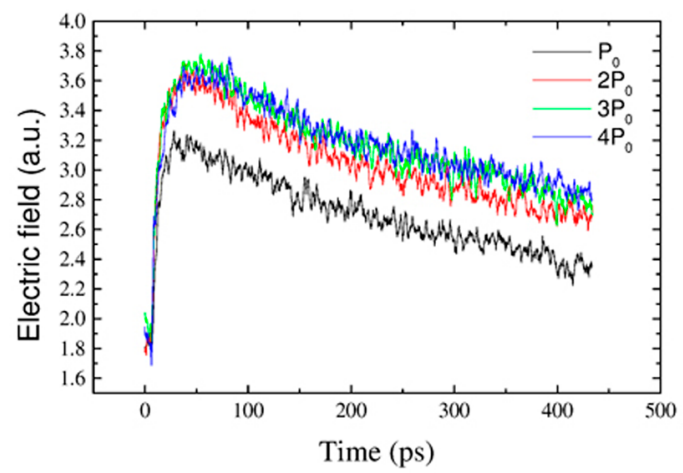

Fig. 4. The measured $\mathrm{THz}$ field as a function of time delay relative to a pump pulse $\left(P_{0}=10 \mathrm{~mW}\right)$. of $\mathrm{THz}$ radiation can occur due to several reasons. One of them may be the growth of the electron momentum relaxation time, that is the increase of the mobility of the charge carriers. Another cause can be determined by increasing the lifetime of non-equilibrium charge carriers. To clarify the nature of this phenomenon, we measured the terahertz pulse waveforms using the electro-optic sampling. Figure 5 shows two waveforms of the terahertz pulse: the first - in the absence of excitation of the first optical pulse, and the second - for the time delay of $30 \mathrm{ps}$ of the first optical pulse with respect to the second one when there is maximum effi-

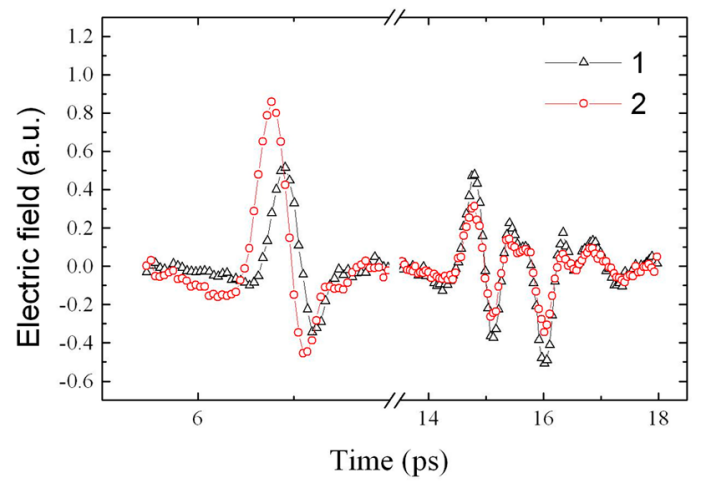

Fig. 5. Waveforms of the THz pulse: 1 no excitement of electron-hole plasma by the first optical pulse, 2 second optical pulse is delayed relative to the first pulse at $30 \mathrm{ps}$.

ciency of THz generation. Also in Fig. 5 we may see the $\mathrm{THz}$ pulse is associated with the reflection of terahertz radiation from the rear surface of the sample. We see that the creation of an additional electron-hole plasma shifts the main $\mathrm{THz}$ pulse in time by about $140 \mathrm{fs}$ in the negative region in relation to the terahertz pulse generated without creating this electron-hole plasma and reflected $\mathrm{THz}$ pulse shifts to the positive region of the timeline. Note that a detailed study of the shifting of $\mathrm{THz}$ pulse waveforms shows an increase of the shift with an increase in the time delay of the first optical pulse with respect to the second one (to a value of about $30 \mathrm{ps}$ ) and its subsequent reduction. This change in the phase of $\mathrm{THz}$ pulse cannot be explained by the characteristic changes of the phase during the process of generation, which is positive as there are a growth of the lifetime of charge carriers and a decrease of their frequency of collisions [ [ 4 ]. This result can be explained if we take into account the characteristic changes of the phase in transmission and reflection $\mathrm{THz}$ pulse through the border between the absorbing and nonabsorbing medium. Indeed, passing of monochromatic plane electromagnetic waves through such a boundary changes the phase of the wave $\varphi$ 


$$
\operatorname{tg} \varphi \approx-\frac{\alpha c \cos \theta}{2 \omega(1+n \cos \theta)},
$$

wherein $\alpha$ is the coefficient of absorption of $\mathrm{THz}$ waves with frequency $\omega, n$ is the refractive index, $\theta$ is the exit angle of the electromagnetic wave in a non-absorbing medium, and $c$ is velocity of light in vacuum. Equation (1) was obtained in the approximation of $\frac{\alpha c}{2 \omega}<n$. From (1) it is clear that the phase due to the absorption of $\mathrm{THz}$ waves of electron-hole plasma has a negative value and is determined by the values a and $n$, which are dependent on the electron momentum relaxation time and the carrier concentration. It can be shown that the absorption coefficient increases, and the refractive index decreases with the increase of the electron momentum relaxation time $\omega<1 / \tau_{\mathrm{p}}$ provided that it is performed for the given experimental conditions. Thus, the experimental results (increase of the efficiency of generation of $\mathrm{THz}$ radiation, phase change of the $\mathrm{THz}$ pulse) can be explained if one takes into account the first reason discussed above, specifically, an increase in the electron momentum relaxation time.

Shifts of $\mathrm{THz}$ waveform by excitation of a pump impulse were determined for samples with NWs of different diameters and AlGaAs-passivated GaAs

(a)

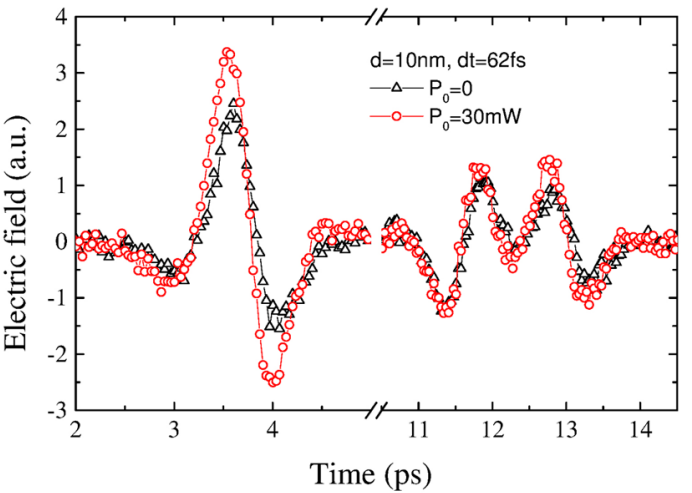

(c)

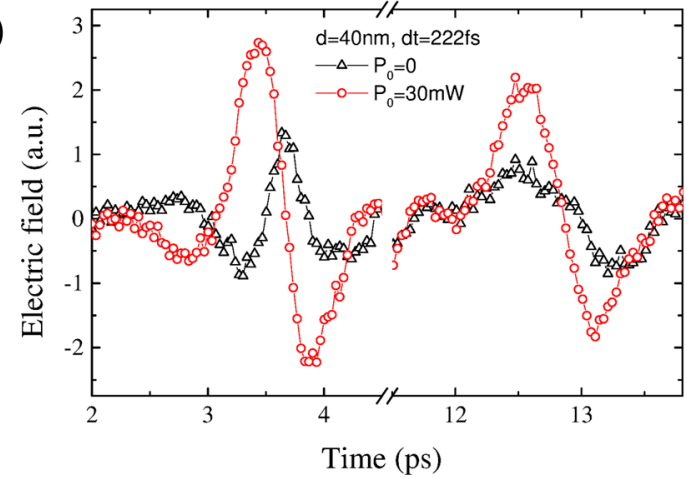

NWs. Figure $6(\mathrm{a}-\mathrm{d})$ shows experimental results: Fig. 6(a-c) for NWs with diameters of 10, 20, and $40 \mathrm{~nm}$, respectively, and Fig. 6(d) for NWs with a diameter of $40 \mathrm{~nm}$ coated with a layer of AlGaAs. From these results it follows that the efficiency of generating of $\mathrm{THz}$ radiation is bigger for the samples with the NWs having a diameter of $10 \mathrm{~nm}$ and AlGaAs-passivated GaAs NWs (Fig. 6(a, d)). Shifts of the waveforms for these samples, on the contrary, are smaller. As already noted, an increase in the efficiency of $\mathrm{THz}$ generation is due to increased electron momentum relaxation time, and this is apparently due to carrier trapping by the ionized scattering centres, which depending on the filling degree will affect the mobility of charge carriers. The results (Fig. $6(\mathrm{a}, \mathrm{d})$ ) suggest the presence of non-radiative centres [ [- $]$ ] in the bulk NWs and on the surface (Fig. $6(b, c))$. The similarity of the results for the sample with NWs having a diameter of $10 \mathrm{~nm}$ and the sample with AlGaAs-passivated GaAs NWs (for which the concentration of surface states is lower than for the uncoated one) indicates one-dimensional electron transport in NWs of small diameter $(10 \mathrm{~nm})$ at room temperature.

Further regeneration of $\mathrm{THz}$ emission is a consequence of carrier retrapping and radiative recombination.

(b)

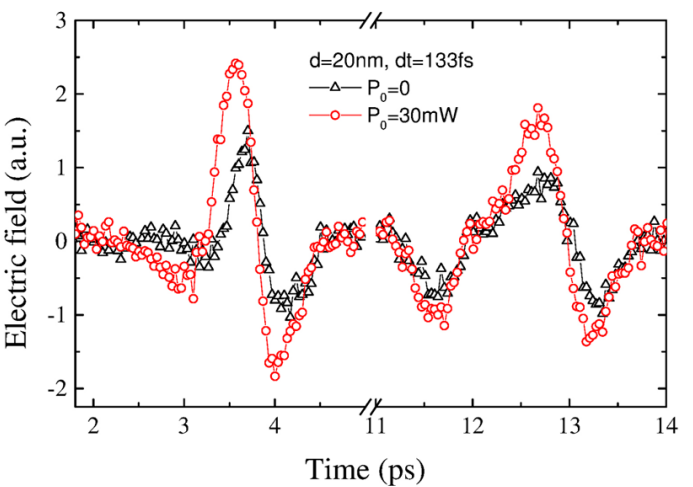

(d)

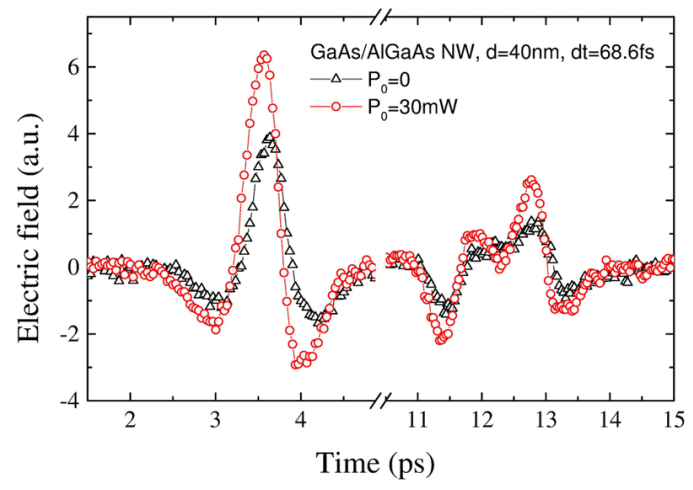

Fig. 6. Waveforms of the Thz pulse for (a) GaAs NWs, shift $62 \mathrm{fs}$, (b) GaAs NWs, shift $133 \mathrm{fs}$, (c) GaAs NWs shift 222 fs, and (d) AlGaAs-passivated GaAs NWs, shift 68.6 fs. 


\section{Acknowledgements}

The study was supported by The Ministry of Education and Science of Russian Federation, project 14.B37.21.1617, and the RFBR program.

\section{References}

[1] S.O. Kognovitskii, V.V. Travnikov, Ya. Aaviksoo, and I. Reimand, Phys. Solid State 39, 907-912 (1997).

\section{LABAI SPARTI KRŪVININKŲ DINAMIKA GaAs NANOGIJOSE}

\author{
V.N. Trukhin a,c, A.D. Buravlev a,b, V. Dhaka ${ }^{\text {d }}$, G.E. Cirlin ${ }^{\text {a,b }}$, I.A. Mustafin ${ }^{\mathrm{a}, \mathrm{c}}$, \\ M.A. Kaliteevski ${ }^{\text {a,b }}$, H. Lipsanen ${ }^{\mathrm{d}}$, Yu.B. Samsonenko ${ }^{\mathrm{a}, \mathrm{b}}$ \\ ${ }^{a}$ Jofés fizikos technikos institutas, Sankt Peterburgas, Rusija \\ ${ }^{\mathrm{b}}$ Sankt Peterburgo akademinis universitetas, Sankt Peterburgas, Rusija \\ c Sankt Peterburgo valstybinis informaciniu technologiju, mechanikos ir optikos universitetas, Sankt Peterburgas, Rusija \\ ${ }^{\mathrm{A}}$ Aalto universitetas, Espas, Suomija
}

